\title{
PyFuncover: full proteome search for a specific function using BLAST and PFAM
}

\author{
Yoan Bouzin ${ }^{1}$, Benjamin Thomas Viart ${ }^{1}$, María Moriel-Carretero ${ }^{2}$, Sofia Kossida ${ }^{1 凶}$ \\ ${ }^{1}$ IMGT $^{\circledR}$, IGH, Univ Montpellier, CNRS, Montpellier, France \\ ${ }^{2}$ CRBM, Univ Montpellier, CNRS, Montpellier, France \\ Competing interests: YB none; BTV none; MMC none; SK none
}

\section{Abstract}

Python Function uncover (PyFuncover) is a new bioinformatic tool able to search proteins with a specific function in a full proteome. The pipeline coded in python uses BLAST alignment and the sequences from a PFAM family as the search seed. We tested PyFuncover using the fatty acid-binding family (FABP) Lipocalin_7 from PFAM (version 32.0, September 2018) against the Homo sapiens NCBI proteome. After applying the scoring function in all the BLAST results, the data were classified and submitted to a GO-TERM analysis using bioDBnet. Analyses showed that all families of FABPs were ranked within the top scores. Included within this category were also families able to bind to hydrophobic molecules similar to fatty acids such as the retinol acid transporter and the cellular retinoic acid-binding protein.

Availability: PyFuncover source code is freely available at https://github.com/Tuisto59/PyFuncover/ under the GPL licence.

\section{Introduction}

High-throughput technologies produce massive amount of data and bioinformatics approaches help predict and annotate protein function using increasingly complex and precise methods. One example is the NCBI annotation pipeline (Thibaud-Nissen et al., 2016). The human genome sequence was released in 2003 but the annotation of the human proteome in January of 2018 (GRCh38.p12) still contains 2,404 uncharacterised proteins (out of 113,620). Protein families for which the relationship between sequence and function is more complex pose the most significant challenges. The enzymes are particularly tricky because only a small part of the protein is responsible for its function. Moreover, specific binding motifs for which knowledge is still partial and poorly annotated add up to this category.

In 2011 a tool called Ada-BLAST was published and used to predict a fatty acid-binding motif in the human protein BRCA1 (Hedgepeth et al., 2015) and the horse Oxy-myoglobin (Patterson et al., 2011), revealing in those already well-known proteins a new property. Today, this tool is no longer available. Inspired by the methodology explained in (Hong et al., 2009; Patterson et al., 2011; Dae Ko et al., 2011; Hedgepeth et al., 2015; Chintapalli et al., 2015), we created PyFuncover.

PyFuncover is a pipeline able to rank each protein from a proteome according to a specific Protein FAMily

\section{Article history}

Received: 04 February 2019

Accepted: 02 March 2019

Published: 25 April 2019
(PFAM) (El-Gebali et al., 2019). As a proof of concept, we used this tool to find proteins with putative fatty acidbinding property in the human proteome. We used as a seed the Lipocalin_7 domain family (PF14651 $\left.{ }^{1}\right)$.

\section{Workflow}

To study a specific activity and to identify other proteins with potentially similar function, the first step is to recover a large set of protein sequences using as a seed the protein annotated with the desired function. Each chosen sequence will make ten iterations (PSI-BLAST accepts a list of multiple sequences, but only the first sequences are used) (see Figure 1, blue box).

Specific family sequences can be downloaded from the PFAM database as a multiple sequences alignment (MSA) from $\mathrm{NCBI}^{2}$ or UniProt ${ }^{3}$ using various formats. Each sequence has a header containing the protein accession, followed by a slash and the domain boundary. The accession of all PSI-BLAST reports is compiled, and each PFAM accession is checked if it is included in the PSI-BLAST results. Other sequences can be from a close family to the chosen one or belong to the same PFAM family. Gaps from the MSA are removed (see Figure 1, green boxes), and a BLAST database is made (see Figure 1, black box).

A whole proteome dataset can be downloaded or any set of proteins in FASTA format (see Figure 1, orange

\footnotetext{
${ }^{1}$ https://pfam.xfam.org/family/Lipocalin_7

${ }^{2}$ https://www.ncbi.nlm.nih.gov/

${ }^{3}$ https://www.uniprot.org/
}

(c) 2019 Bouzin et al.; the authors have retained copyright and granted the Journal right of first publication; the work has been simultaneously released under a Creative Commons Attribution Licence, which allows others to share the work, while acknowledging the original authorship and initial publication in this Journal. The full licence notice is available at http://journal.embnet.org. 


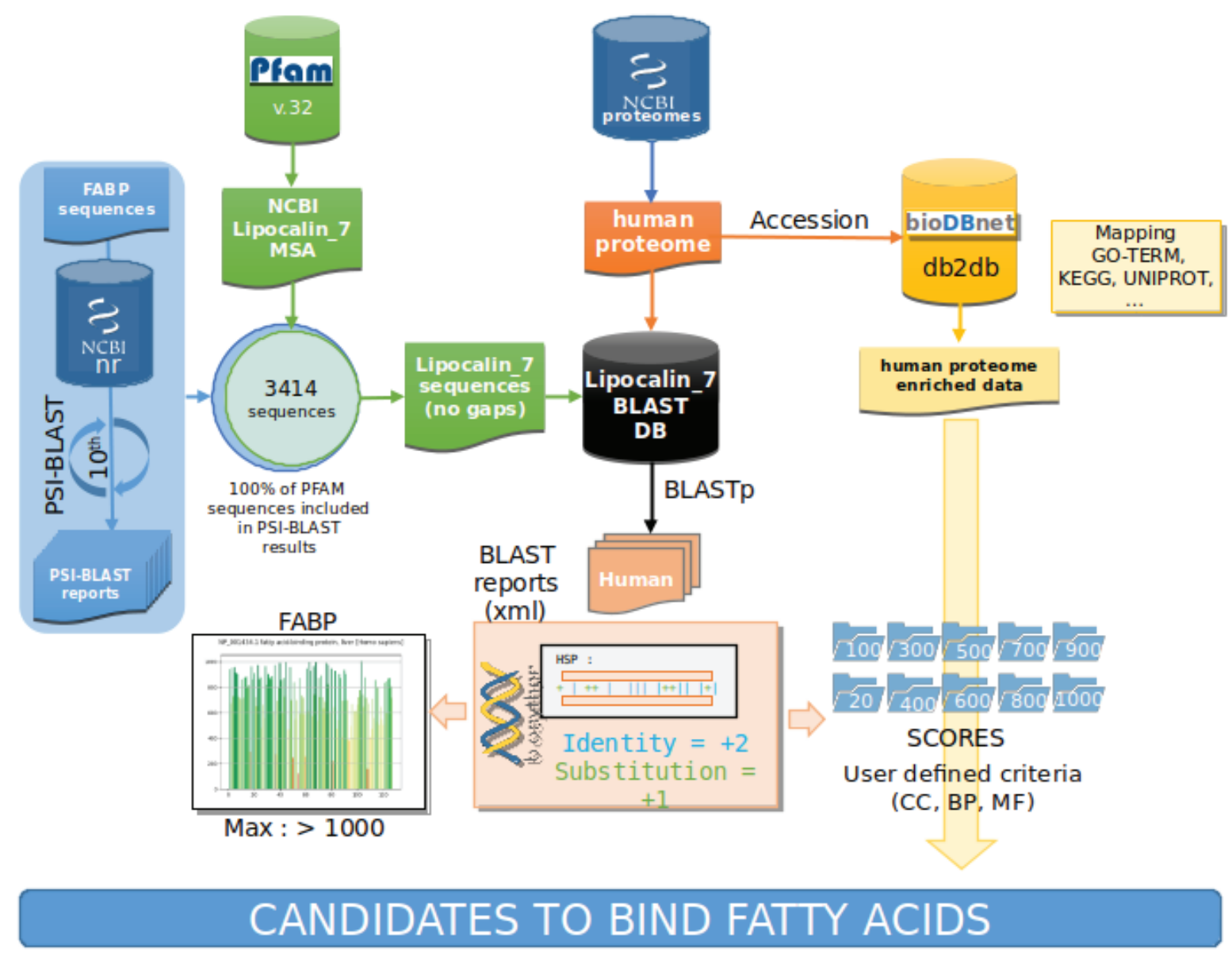

Figure 1. Workflow of PyFuncover.

box). For each sequence, a BLASTp is performed against the PFAM BLAST DB (see Figure 1, black box). For each protein (subject) that matches our sequence (query), BLAST produces alignments, called High Scoring Pairs (HSPs). A score of two, for all the identities, and a score of one, for all positive substitutions, is computed for each alignment. Accession numbers from NCBI are used to retrieve data from different databases (GO-Terms, UniProt, KEGG, PDB, BioCyc, Ensembl, GenBank...) (Ashburner et al., 2000; Berman et al., 2000; Clark et al. , 2016; Kanehisa et al., 2019; Karp et al., 2017; UniProt Consortium, 2018; Zerbino et al., 2018) using the crossreference database web application BioDBnet (db2db) (Mudunuri et al., 2009) and compiled into a biologistfriendly table. This makes the results easy to open and parse using a spreadsheet software such as Excel.

\section{Proof of concept}

To test PyFuncover, we used a selection of human Fatty Acid-Binding Proteins (FABPs) (Table 1). The FABPs are part of the lipocalin_7 family (PF14651). The accession numbers of the 3414 sequences from the MSA of NCBI were compared with all the PSI-BLAST results. All the sequences were included into the PSI-BLAST results, and MSA were used to make the BLAST database. Using CDD-Search (Marchler-Bauer et al., 2017), we checked the accessions of the PSI-BLAST reports. The accessions

corresponded to the PFAM Lipocalin_7 or to the lipocalins 4 and 5 as expected since all three are members of the Calycin superfamily. The human proteome was downloaded to perform a BLASTp against the database made from the MSA. The XML reports were parsed using BioPython (Cock et al., 2009).

Each amino-acid of each protein obtains a score. Scores can be represented as a barplot for visual analysis (Figure 2). Proteins were split into ten folders (from 100 up to 1000) based on its highest scored amino acid (Figure 2). For the FABPs input set, the highest score was 1052 for FABP7 (isoform X4, NP_001305971). Human

Table 1. List of the FABP used for the PSI-BLAST run.

\begin{tabular}{ll} 
FABP & UNIPROT Accession \\
\hline FABP1 & P07148 \\
\hline FABP2 & P12104 \\
\hline FABP3 (FABP11) & P05413 \\
\hline FABP4 & P15090 \\
\hline FABP5 & Q01469 \\
\hline FABP6 & P51161 \\
\hline FABP7 & O15540 \\
\hline FABP8 (PMP2) & P02689 \\
\hline FABP9 & Q0Z7S8 \\
\hline FABP12 & A6NFH5
\end{tabular}




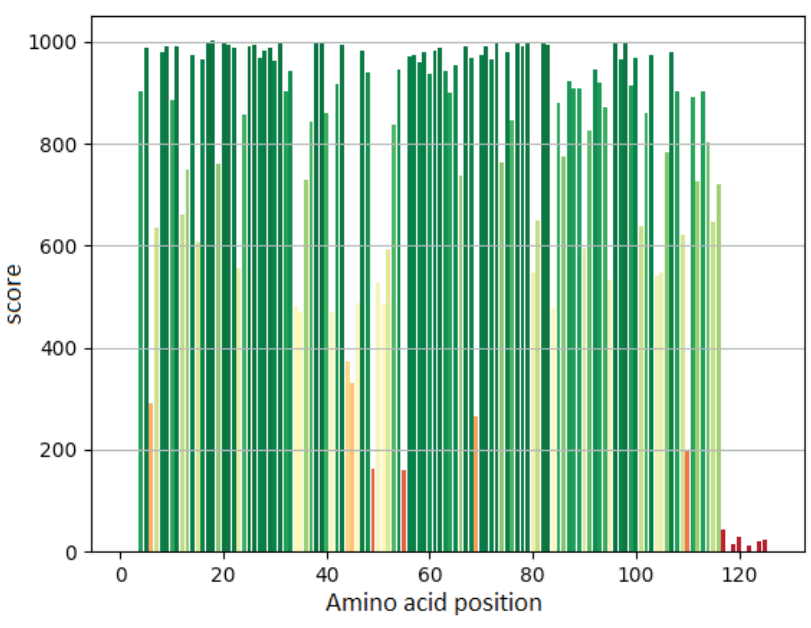

XP_011539309.1 fatty acid-binding protein, heart isoform X1 [Homo sapiens]

Figure 2. Score histogram per amino acid along the sequence of the FABP1 (isoform X1) of Homo sapiens. Colour range stands from less in red to high in green.

proteomes accession numbers were crossed with the GO-TERM database, using BioDBnet (see Figure 1, yellow box).

Considering the proteins with a score above 900 (arbitrarily chosen), we found members of all the nine FABPs families (Table 2). Above this threshold, we also found five (Cellular) Retinol-Binding Proteins (CRBPs) and two (Cellular) Retinoic Acid-Binding Proteins (CRABPs). This is remarkable, because FABPs, CRBPs and CRABPs are all three subfamilies of the intracellular Lipid-Binding Proteins (iLBPs) family. Moreover both retinol and retinoic acid display a partially similar structure to that of fatty acids (Smathers and Petersen, 2011). As expected the FABP1 family is ranked first using highest mean amino-acid score reaching 735 (Figure 3).

\section{Conclusions}

The dataset with a score above 900 contains the top one per cent of the input or 1,530 proteins. This number dramatically exceeds that described above as a proof of concept. This tool aims at helping biologists investigate their favourite set of proteins with a simple sequencefunction scoring method. PyFuncover output table combines protein identification, score and several useful

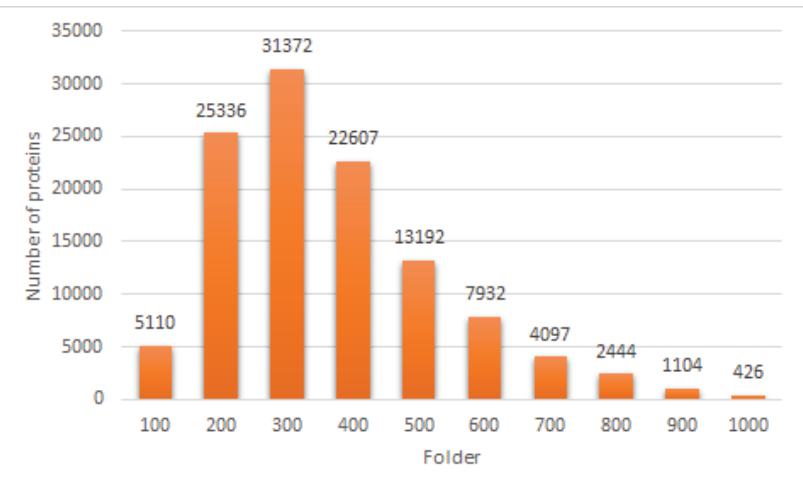

Figure 3. Number of protein in each folder.
Table 2. Proteins implicated in the binding of fatty acids and related hydrophobic molecules from Homo sapiens found in the 900 and 1000 folders.

\begin{tabular}{lll} 
Protein & Score $\mathbf{1 0 0 0}$ & Score 900 \\
\hline FABPs & FABP1, 3, 7, 8, 12 & FABPS2, 3, 4, 5, 6, 7, 9 \\
\hline RBPs & RBP1, 5, 7 & RPB2, 5 \\
\hline CRABPs & & CRABP1, 2
\end{tabular}

databases cross-references for handy investigation. Additionally, while we used it here to detect putative fatty acids-binding motifs, PyFuncover can be tailored to search other functional features matching the user's wishes.

\section{Key Points}

- PyFuncover is a new bioinformatic tool to search proteins with a specific function in a full proteome.

- Using the Lipocalin 7 family as input we observed in the top-ranked proteins all families of FABPs as well as families able to bind to hydrophobic molecules similar to fatty acids.

- PyFuncover output table combines protein identification, score and several useful databases cross-references for handy investigation.

- This tool aims at helping biologists investigate their favorite set of proteins with a simple sequencefunction scoring method.

\section{Acknowledements}

This work was supported by Merck Sharp and Dohme Avenir (GnoSTic) to S. Kossida and by the ATIP-Avenir program to M. Moriel-Carretero.

\section{References}

1. Ashburner M, Ball CA, Blake JA, Botstein D, Butler H, et al. (2000) Gene ontology: tool for the unification of biology. The Gene Ontology Consortium. 25 (1), 25-29. http://dx.doi. org/10.1038/75556

2. Berman HM, Westbrook J, Feng Z, Gilliland G, Bhat TN, et al. (2000) The Protein Data Bank. Nucleic Acids Res. 28 (1), 235242. http://dx.doi.org/10.1093/nar/28.1.235

3. Chintapalli SV, Bhardwaj G, Patel R, Shah N, Patterson RL, et al. (2015) Molecular dynamic simulations reveal the structural determinants of Fatty Acid binding to oxy-myoglobin. PLoS One 10 (6), e0128496. http://dx.doi.org/10.1371/journal. pone.0128496

4. Clark K, Karsch-Mizrachi I, Lipman DJ, Ostell J, and Sayers EW (2016) GenBank. Nucleic Acids Res. 44 (D1), D67-72. http:// dx.doi.org/10.1093/nar/gkw1070

5. Cock PJA, Antao T, Chang JT, Chapman BA, Cox CJ, et al. (2009) Biopython: freely available Python tools for computational molecular biology and bioinformatics. Bioinformatics 25 (11), 1422-1423. http://dx.doi.org/10.1093/bioinformatics/btp163

6. El-Gebali S, Mistry J, Bateman A, Eddy SR, Luciani A, et al. (2019) The Pfam protein families database in 2019. Nucleic Acids Res. 47 (D1), D427-D432. http://dx.doi.org/10.1093/nar/gky995

7. Hedgepeth SC, Garcia MI, Wagner LE 2nd, Rodriguez AM, Chintapalli SV, et al. (2015) The BRCA1 tumor suppressor binds to inositol 1,4,5-trisphosphate receptors to stimulate apoptotic 
calcium release. J. Biol. Chem. 290 (11), 7304-7313. http://dx.doi org $/ 10.1074 /$ jbc.M114.611186

8. Hong Y, Chalkia D, Ko KD, Bhardwaj G, Chang GS, et al (2009) Phylogenetic Profiles Reveal Structural and Functional Determinants of Lipid-binding. J. Proteomics Bioinform. 2, 139149. http://dx.doi.org/10.4172/jpb.1000071

9. Kanehisa M, Sato Y, Furumichi M, Morishima K, and Tanabe M (2019) New approach for understanding genome variations in KEGG. Nucleic Acids Res. 47 (D1), D590-D595. http://dx.doi. org/10.1093/nar/gky962

10. Karp PD, Billington R, Caspi R, Fulcher CA, Latendresse M, et al. (2017) The BioCyc collection of microbial genomes and metabolic pathways. Brief. Bioinform. http://dx.doi.org/10.1093/ $\mathrm{bib} / \mathrm{bbx} 085$

11. Kyung Dae Ko, Chunmei Liu, Rwebangira MR, Burge L, and Southerland W (2011) The development of a proteomic analyzing pipeline to identify proteins with multiple RRMs and predict their domain boundaries. In: 2011 IEEE International Conference on Bioinformatics and Biomedicine Workshops (BIBMW). IEEE, pp. 374-381 http://dx.doi.org/10.1109/BIBMW.2011.6112401.

12. Marchler-Bauer A, Bo Y, Han L, He J, Lanczycki CJ, et al (2017) CDD/SPARCLE: functional classification of proteins via subfamily domain architectures. Nucleic Acids Res. 45 (D1), D200-D203. http://dx.doi.org/10.1093/nar/gkw1129

13. Mudunuri U, Che A, Yi M, and Stephens RM (2009) bioDBnet: the biological database network. Bioinformatics 25 (4), 555-556. http://dx.doi.org/10.1093/bioinformatics/btn654

14. Patterson RL, Hong Y, Chintapalli SV, Bhardwaj G, Zhang Z, et al. (2011) Adaptive-BLAST: A User-defined Platform for the Study of Proteins. J. Integr. OMICS 1 (1) http://dx.doi.org/10.5584/ jiomics.v1i1.33

15. Smathers RL and Petersen DR (2011) The human fatty acidbinding protein family: Evolutionary divergences and functions. Hum. Genomics 5 (3), 170. http://dx.doi.org/10.1186/1479-7364$5-3-170$

16. Thibaud-Nissen F, DiCuccio M, Hlavina W, Kimchi A, Kitts PA, et al. (2016) P8008 The NCBI Eukaryotic Genome Annotation Pipeline. J. Anim. Sci. 94 (suppl_4), 184-184.

17. UniProt Consortium $\mathrm{T}$ (2018) UniProt: the universal protein knowledgebase. Nucleic Acids Res. 46 (5), 2699. http://dx.doi. org/10.1093/nar/gkv1189

18. Zerbino DR, Achuthan P, Akanni W, Amode MR, Barrell D, et al. (2018) Ensembl 2018. Nucleic Acids Res. 46 (D1), D754-D761. http://dx.doi.org/10.1093/nar/gkx1098 